\title{
ISOLAMENTO E ATIVIDADES BIOLÓGICAS DE PRODUTOS NATURAIS DAS ESPONJAS Monanchora arbuscula, Aplysina sp., Petromica ciocalyptoides E Topsentia ophiraphidites, DA ASCÍDIA Didemnum ligulum E DO OCTOCORAL Carijoa riisei
}

\author{
Miriam H. Kossuga, Simone P. de Lira, Andréa M. Nascimento, Maria Teresa P. Gambardella e Roberto G. S. Berlinck* \\ Instituto de Química de São Carlos, Universidade de São Paulo, CP 780, 13560-970 São Carlos - SP, Brasil \\ Yohandra R. Torres \\ Departamento de Química, Universidade Estadual do Centro-Oeste, Rua Camargo Varela de Sá, 3, 85040-080 Guarapuava - \\ PR, Brasil \\ Gislene G. F. Nascimento \\ Faculdade de Ciências da Saúde, Universidade Metodista de Piracicaba, Rodovia do Açúcar, km 156, 13400-901 Piracicaba - \\ SP, Brasil \\ Eli F. Pimenta, Marcio Silva, Otávio H. Thiemann e Glaucius Oliva \\ Instituto de Física de São Carlos, Universidade de São Paulo, São Carlos - SP, Brasil \\ André G. Tempone e Márcia S. C. Melhem \\ Divisão de Biologia Médica, Instituto Adolfo Lutz, Av. Dr. Arnaldo, 351, São Paulo - SP, Brasil \\ Ana O. de Souza, Fabio C. S. Galetti e Célio L. Silva \\ Departamento de Bioquímica e Imunologia, Faculdade de Medicina de Ribeirão Preto, Universidade de São Paulo, Ribeirão \\ Preto - SP, Brasil \\ Bruno Cavalcanti, Claudia O. Pessoa e Manoel O. Moraes \\ Departamento de Fisiologia e Farmacologia, Faculdade de Medicina, Universidade Federal do Ceará, Fortaleza - CE, Brasil \\ Eduardo Hajdu \\ Museu Nacional, Universidade Federal do Rio de Janeiro, Quinta da Boa Vista, s/n, 20940-040 Rio de Janeiro - RJ, Brasil \\ Solange Peixinho \\ Departamento de Biologia, Universidade Federal da Bahia, Salvador - BA, Brasil \\ Rosana M. Rocha \\ Departamento de Zoologia, Setor de Ciências Biológicas, Universidade Federal do Paraná, CP 19020, 81531-990 Curitiba - PR, \\ Brasil \\ Recebido em 13/7/06; aceito em 18/1/07; publicado na web em 24/7/07
}

\begin{abstract}
ISOLATION AND BIOLOGICAL ACTIVITIES OF SECONDARY METABOLITES FROM THE SPONGES Monanchora aff. arbuscula, Aplysina sp. Petromica ciocalyptoides AND Topsentia ophiraphidites, FROM THE ASCIDIAN Didemnum ligulum AND FROM THE OCTOCORAL Carijoa riisei. The investigation of extracts from six species of marine invertebrates yielded one new and several known natural products. Isoptilocaulin from the sponge Monanchora aff. arbuscula displayed antimicrobial activity at $1.3 \mu \mathrm{g} / \mathrm{mL}$ against an oxacillin-resistant strain of Staphylococcus aureus. Five inactive known dibromotyrosine derivatives, $\mathbf{2}$ - 6, were isolated from a new species of marine sponge, Aplysina sp. The sponges Petromica ciocalyptoides and Topsentia ophiraphidites yielded the known halistanol sulfate A (7) as an inhibitor of the antileishmanial target adenosine phosphoribosyl transferase. The ascidian Didemnum ligulum yielded asterubin (10) and the new $N, N$-dimethyl- $O$-methylethanolamine (11). The octocoral Carijoa riisei yielded the known 18-acetoxypregna1,4,20-trien-3-one (12), which displayed cytotoxic activity against the cancer cell lines SF295, MDA-MB435, HCT8 and HL60.
\end{abstract}

Keywords: marine sponge; ascidian; octocoral.

\section{INTRODUÇÃO}

Nos últimos anos, investigações químicas e farmacológicas de organismos marinhos têm contribuído significativamente para a descoberta de novas substâncias químicas potencialmente bioativas para fins terapêuticos ${ }^{1}$. Embora o litoral brasileiro seja o segundo mais extenso depois da Austrália, o desenvolvimento da química de produtos naturais de organismos marinhos no Brasil foi negligenciado por muitos anos, porque o principal foco da química de produtos naturais brasileira foi direcionado ao estudo de plantas. Desta maneira, a fauna marinha brasileira ainda é pouco explorada na pesquisa por produtos naturais biologicamente ativos, estrutu-

*e-mail: rgsberlinck@iqsc.usp.br ralmente inéditos ou taxonomicamente relevantes ${ }^{2}$.

No presente trabalho relata-se a investigação química dos extratos brutos bioativos de quatro esponjas, uma ascídia e um octocoral. Descreve-se o isolamento de um alcalóide guanidínico a partir da esponja Monanchora aff. arbuscula, de cinco derivados da dibromotirosina, a partir da esponja Aplysina sp., o isolamento do mesmo esterol trissulfatado a partir de ambas esponjas Petromica ciocalyptoides e Topsentia ophiraphidites, o primeiro isolamento da asterubina, a partir de uma ascídia, Didemnum ligulum, da qual também foi isolada a $N$ - $N$-dimetil- $O$-metiletanoamina, inédito na literatura, além do 18-acetoxipregna-1,4,20-trien-3-ona a partir do octocoral Carijoa riisei. Também são apresentados os resultados de testes de atividade biológica realizados com estes metabólitos secundários. 


\section{RESULTADOS E DISCUSSÃO}

\section{Investigação química da esponja Monanchora arbuscula}

O extrato metanólico da esponja Monanchora arbuscula apresentou potentes atividades antibacteriana, antimicobacteriana contra Mycobacterium tuberculosis H37Rv e citotóxica. O extrato metanólico foi então submetido a várias separações cromatográficas (ver Parte Experimental), resultando na obtenção do alcalóide guanidínico isoptilocaulina (1). A análise do espectro de RMN de ${ }^{13} \mathrm{C}$ e experimento DEPT $135^{\circ}$ de $\mathbf{1}\left(\mathrm{MeOH}-d_{4}, 125 \mathrm{MHz}\right)$ permitiu observar quinze sinais referentes a dois grupos metila, seis grupos metileno (dois dos quais com deslocamentos químicos idênticos), quatro grupos metino e três carbonos do tipo $\mathrm{sp}^{2}$. O sinal em $\delta$ 156,4 foi atribuído ao carbono da ligação dupla carbono-nitrogênio guanidínico e os dois sinais em $\delta$ 136,3 e 132,9 foram atribuídos a dois carbonos de uma ligação dupla carbono-carbono. A análise conjunta dos espectros de $\mathrm{RMN}$ de ${ }^{1} \mathrm{H}\left(\mathrm{MeOH}-d_{4}, 500 \mathrm{MHz}\right)$, de ${ }^{13} \mathrm{C}$ e bidimensionais (HMQC, HMBC e COSY), espectro de massas, bem como a comparação com dados da literatura ${ }^{3,4}$ permitiram identificar a substância $\mathbf{1}$ como sendo a isoptilocaulina.

Estudos químicos anteriores realizados com esponjas do gênero Monanchora demonstraram que espécies deste gênero são uma rica fonte de alcalóides guanidínicos ${ }^{5}$. Recentemente, a isoptilocaulina foi isolada de uma esponja do gênero Monanchora (cf. Monanchora unguifera $)^{4}$. Todavia, considerando-se que os gêneros Ptilocaulis, Monanchora e Crambe possam ser considerados um único gênero, Crambe ${ }^{6}$, é possível que o isolamento da isoptilocaulina (1) de $M$. unguifera esteja relacionada à sua ocorrência em diferentes espécies de esponjas de um mesmo gênero. A isoptilocaulina (1) foi submetida à avaliação de sua atividade antibacteriana contra linhagens suscetíveis e resistentes a antibióticos de diferentes bactérias (Tabela 1), apresentando concentração inibitória mínima (CIM) de $170 \mu \mathrm{g} / \mathrm{mL}$ contra Staphylococcus aureus ATCC 25923 (cloranfenicol: 1,3 $\mu \mathrm{g} / \mathrm{mL}$ ), $85 \mu \mathrm{g} / \mathrm{mL}$ contra Escherichia coli ATCC 25922 (cloranfenicol: $20 \mu \mathrm{g} / \mathrm{mL}$ ), $170 \mu \mathrm{g} /$ $\mathrm{mL}$ contra Pseudomonas aeruginosa ATCC 27583 (cloranfenicol: $13 \mu \mathrm{g} / \mathrm{mL}$ ), e CIM de 1,3 , e $85,0 \mu \mathrm{g} / \mathrm{mL}$ contra duas linhagens de $S$. aureus resistentes ao antibiótico oxacilina, contra as quais o antibiótico padrão cloranfenicol não foi ativo. Sendo assim, a isoptilocaulina (1) mostrou ser um potente antibiótico contra linhagens resistentes de $S$. aureus, causadoras de infecções hospitalares para as quais existem poucos antibióticos efetivos. Este é o primeiro estudo da atividade antibacteriana da isoptilocaulina contra linhagens resistentes de bactérias.

\section{Investigação química da esponja Aplysina sp.}

O gênero Aplysina pertence à ordem Verongida, conhecido como sendo fonte de substâncias bromadas derivadas da tirosina ${ }^{7}$. Espécimens de Aplysina sp., recentemente descrita como sendo uma espécie nova de esponja do litoral baiano ${ }^{8}$, foram obtidos na Baía de Todos os Santos, Salvador (BA) em 1999. A esponja preservada em EtOH foi diretamente enviada ao Museu Nacional (UFRJ), onde foi identificada. O material biológico foi separado do $\mathrm{EtOH}$, triturado em $\mathrm{MeOH}$, e os extratos etanólico e metanólico foram reunidos e evaporados. Em seguida, o extrato hidroalcoólico foi submetido a uma série de partições. A fração solúvel em AcOEt foi então submetida a uma série de separações cromatográficas, (ver Parte Experimental), resultando no isolamento de cinco substâncias (2-6). A substância 2 mostrou ser um sólido vítreo, cujo espectro de $\mathrm{RMN}$ de ${ }^{1} \mathrm{H}\left(\mathrm{CDCl}_{3}, 400 \mathrm{MHz}\right)$ apresentou sinais correspondentes a um grupo metoxila em $\delta 3,13(\mathrm{~s}, 3 \mathrm{H})$, a um grupo etoxila em $\delta 3,35(\mathrm{q}, J=7,0 \mathrm{~Hz}, 2 \mathrm{H})$ e $\delta 1,20(\mathrm{t}, 3 \mathrm{H}, J=7,0 \mathrm{~Hz}$, $3 \mathrm{H}$ ), um sinal em $\delta 6,75(\mathrm{~s}, 2 \mathrm{H})$ relativo a dois hidrogênios olefínicos quimicamente equivalentes e um sinal em $\delta 2,54$ (s, $2 \mathrm{H})$ pertencente a um grupo metileno. A análise conjunta dos espectros de $\mathrm{RMN}$ de ${ }^{1} \mathrm{H}$, de ${ }^{13} \mathrm{C}\left(\mathrm{CDCl}_{3}, 100 \mathrm{MHz}\right)$ e bidimensionais (HMQC, HMBC e COSY) e a comparação com dados da literatura ${ }^{9}$ permitiram identificar a substância 2 como sendo a (3',5'-dibromo-4' -etoxi-1' '-hidroxi-4'-metoxicicloexa-2', 5' dienila) acetamida, a qual mostrou ser essencialmente inativa em ensaios de atividade antimicrobiana contra S. aureus ATCC 25923, E. coli ATCC 25922, P. aeruginosa ATCC 27853, Candida albicans ATCC 10231 e linhagens resistentes à oxacilina de $S$. aureus e $P$. aeruginosa (Tabela 1).

$\mathrm{O}$ espectro de RMN de ${ }^{1} \mathrm{H}$ de $3\left(\mathrm{MeCN}-d_{3}, 500 \mathrm{MHz}\right)$ apresentou dois dubletos em $\delta 2,84$ e $2,89(1 \mathrm{H}$ cada, $J=17,7 \mathrm{~Hz})$, um singleto de um hidrogênio olefínico em $\delta$ 6,46 $(1 \mathrm{H})$; um singleto em $\delta 5,15(1 \mathrm{H})$ de um hidrogênio ligado a carbono oxigenado e um singleto em $\delta 3,74(3 \mathrm{H})$ de um grupo metoxila. O espectro de RMN de ${ }^{13} \mathrm{C}$ da substância 3 (PND e DEPT $135^{\circ}, \mathrm{MeCN}-d_{3}, 125 \mathrm{MHz}$ ) apresentou 9 átomos de carbono, sendo $\mathrm{CH}_{3}$ em $\delta$ 61,5 referente a um grupo metoxila, $\mathrm{CH}_{2}$ em $\delta 42,2$, dois grupos metino, um sp ${ }^{2}$ em $\delta 135,5$ e um sp ${ }^{3}$ em $\delta 89,1$ de carbono metino oxigenado. Além disso, foram observados sinais de 5 carbonos $\mathrm{sp}^{2}$, sendo um em $\delta$ 173,7 , que foi atribuído a um grupo carbonila de éster, três sinais em $\delta 151,2,119,3,107,6$ atribuídos a carbonos $\mathrm{sp}^{2}$ totalmente substituídos, e um sinal em $\delta 77,0$ de carbono quaternário oxigenado. Após a análise dos dados acima descritos e comparação com dados da literatura ${ }^{10}$, a substância 3 foi identificada como sendo a aeroplisinina-2, previamente isolada das esponjas Aplysina aerophoba, A. fistularis forma fulva e A. archeri $^{10-12}$. O composto 3 também demonstrou ser essencialmente inativo contra $S$. aureus ATCC 25923, E. coli ATCC 25922, P. aeruginosa ATCC 27853, C. albicans ATCC 10231 e linhagens resistentes à oxacilina de $S$. aureus e $P$. aeruginosa (Tabela 1).

$\mathrm{O}$ espectro de $\mathrm{RMN}$ de ${ }^{1} \mathrm{H}$ da substância 4 ( $\mathrm{MeCN}-d_{3}, 500$ $\mathrm{MHz}$ ) apresentou sinais em $\delta 6,41$ (s, 1H, H-5), 4,16 (s, 1H, H-1), $3,70(\mathrm{~s}, 3 \mathrm{H}, \mathrm{MeO})$, além de um sistema $\mathrm{AB}$ em $\delta 3,69$ e $3,06(1 \mathrm{H}$ cada, $J=18,2 \mathrm{~Hz}$ ) característico de um sistema espirocicloexadienilisoxazol, comumente encontrado em muitos outros constituintes químicos de esponjas da ordem Verongida. Os outros sinais de ${ }^{1} \mathrm{H}$ observados referem-se a um grupo etoxila em $\delta 4,29$ (q, $J=7,1 \mathrm{~Hz}, 2 \mathrm{H})$ e $\delta 1,30(\mathrm{t}, J=7,1 \mathrm{~Hz}, 3 \mathrm{H})$. O espectro de RMN de ${ }^{13} \mathrm{C}$ de $4\left(\mathrm{MeCN}-d_{3}, 125 \mathrm{MHz}\right)$ exibiu sinais de dois grupos metila ( $\delta 14,4$ e 60,8$)$, dois grupos metileno ( $\delta 40,0$ e 63,0$)$, dois grupos metino $(\delta 132,3$ e 75,1$)$ e cinco carbonos $\mathrm{sp}^{2}$ e um quaternário $(\delta$ $161,0,153,3,149,0,122,3,113,8$ e 92,6, respectivamente). A análise dos espectros HMQC e HMBC e comparação com dados da literatura ${ }^{13,14}$ permitiram determinar a estrutura de 4 como sendo a 2,4-dibromo-1-hidroxi-3-metoxi-6-oxa-3-azaspiro[4.5]dec-2,4,8trieno-8-carboxilato de etila. Embora esta substância seja inédita na literatura, o fato do ácido correspondente já ter sido isolado de Pseudoceratina $\mathrm{sp.}^{13}$ sugere que esta seja um artefato de isolamento formado pela condensação do ácido correspondente com EtOH durante o processo de armazenamento. $\mathrm{O}$ espectro de dicroísmo circular de 4 apresentou efeito Cotton positivo em $\lambda_{\text {max }} 255$ e 290 $\mathrm{nm}$, indicando configuração absoluta $1(R), 6(S)^{7}$.

As substâncias 5 e $\mathbf{6}$ foram identificadas como sendo a [3,5dibromo-4-[(2-oxo-5-oxazolidinila)]metoxifenil]-2-oxazolidinona ${ }^{15}$ e o 2-(3,5-dibromo-4-metoxifenil)- $N, N, N$-trimetiletanamônio ${ }^{16}$. O composto 5 também não apresentou atividade antimicrobiana contra os mesmos microrganismos patogênicos supra mencionados (Tabela 1). Este é o primeiro estudo químico realizado com a esponja Aplysina sp.. 

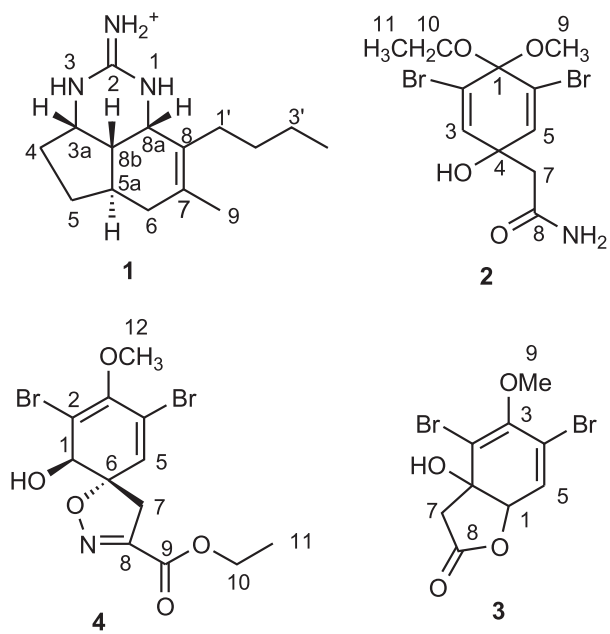<smiles>COC1=C(Br)C2(O)C(=O)O[C@@H]2C=C1Br</smiles><smiles>O=C1NCC(COc2c(Br)cc(C3CNC(=O)O3)cc2Br)O1</smiles><smiles>COc1c(Br)cc(CC[N+](C)(C)C)cc1Br</smiles>

6

Tabela 1. Resultados da atividade antimicrobiana in vitro das substâncias $\mathbf{1}, \mathbf{2}, \mathbf{3}$ e $\mathbf{5}$

\begin{tabular}{lcccccccc}
\hline Substâncias & \multicolumn{8}{c}{ Linhagens Microbianas CIM $(\mu \mathrm{g} / \mathrm{mL})$} \\
\hline & $\mathbf{1}$ & $\mathbf{2}$ & $\mathbf{3}$ & $\mathbf{4}$ & $\mathbf{5}$ & $\mathbf{6}$ & $\mathbf{7}$ & $\mathbf{8}$ \\
$\mathbf{1}$ & 170 & 85 & 170 & 1,3 & 85 & $\mathrm{nt}$ & $\mathrm{nt}$ & $\mathrm{nt}$ \\
$\mathbf{2}$ & 1250 & 1250 & - & - & - & - & - & 625 \\
$\mathbf{3}$ & - & - & - & - & - & 250 & - & - \\
$\mathbf{5}$ & 1250 & 1250 & 1250 & - & - & - & - & 625
\end{tabular}

Linhagens microbianas: 1. Staphylococcus aureus (ATCC 25923) Gram positiva; 2. Escherichia coli (ATCC 25922) - Gram negativa; 3. Pseudomonas aeroginosa (ATCC 27853) - Gram negativa; 4. Staphylococcus aureus ORSA 8; 5. Staphylococcus aureus ORSA 108; 6. P. aeruginosa $13 ; 7$. P. aeruginosa $\mathrm{P} 1 ; 8$. Candida albicans (ATCC 10231) - levedura. ORSA: cepas de Staphylococcus aureus resistentes à oxacilina. (-):ausência de inibição; (nt): não testada.

\section{Investigação química das esponjas Petromica ciocalyptoides e Topsentia ophiraphidites}

O extrato metanólico da esponja Petromica ciocalyptoides coletada na Baía de Todos os Santos (Salvador, BA) apresentou atividade inibitória da enzima adenosina fosforribosil transferase isolada de Leishmania tarantolae ( $98 \%$ de inibição a $50 \mu \mathrm{g} / \mathrm{mL}$ ) e foi submetido a uma série de separações cromatográficas biomonitoradas. $\mathrm{O}$ espectro de $\mathrm{RMN}-{ }^{1} \mathrm{H}\left(\mathrm{MeOH}-d_{4}, 400 \mathrm{MHz}\right)$ do constituinte ativo isolado (7) em MeOH- $d_{4}$ apresentou dois sinais alargados de hidrogênios em $\delta$ 4,80 e 4,75 (sl), sugerindo estarem ligados a carbonos substituídos por grupos sulfato. Outro sinal de hidrogênio oximetínico foi observado em $\delta 4,19$ (dt, 4,4 e 10,9 Hz). Sinais de hidrogênios entre $\delta 2,5$ e 0,5 , incluindo sinais de grupos metila em $\delta 0,69,0,83,0,95,0,85(t$ butila) e 0,83 , sugeriram uma estrutura do tipo esteroidal substituída com grupos hidroxila sulfatados. $\mathrm{O}$ espectro de massas em modo $\mathrm{FAB}^{+}$ de 7 apresentou um pico de íon pseudo-molecular $[\mathrm{M}+\mathrm{Na}]^{+}$de intensidade muito pequena em $\mathrm{m} / z$, 709. A determinação estrutural do esterol trissulfatado 7 foi completada após a obtenção do espectro de massas do derivado dessulfatado e peracetilado $\mathbf{9}$. O composto peracetilado 9 forneceu espectro de massas em modo impacto de elétrons com pico de íon molecular $\mathrm{M}^{+}$em $\mathrm{m} / \mathrm{z}$ 574. Uma medida de massa exata deste forneceu o valor de 574,42324 (calculado: 574,42334, $\Delta \mathrm{mu} 0,1 \mathrm{ppm}$ ), compatível com a fórmula $\mathrm{C}_{35} \mathrm{H}_{58} \mathrm{O}_{6}$. A análise dos espectros de RMN${ }^{1} \mathrm{H}, \mathrm{RMN}-{ }^{13} \mathrm{C}$ (BBD e DEPT, MeOH- $d_{4}, 100 \mathrm{MHz}$ ), HMQC, COSY ${ }^{1} \mathrm{H}-{ }^{1} \mathrm{H}$ e $\mathrm{HMBC}$ e comparação com dados da literatura ${ }^{17}$ indicaram tratar-se do trissulfato de halistanol A (7), originalmente isolado da esponja Halichondria cf. moorei e subseqüentemente de outras espécies de esponjas. O trissulfato de halistanol A (7) isolado da esponja Halichondria cf. moorei inibiu o crescimento de fungos e de bactérias Gram-positivas e Gram-negativas não especificadas ${ }^{17,18}$. Também apresentou atividade hemolítica ${ }^{19}$, atividade inibidora de várias enzimas ${ }^{20-}$ ${ }^{22}$ e atividade "anti-fouling" contra larvas da craca Balanus amphitrite com dose de inibição de $2,9 \mu \mathrm{g} / \mathrm{mL}^{23}$. O trissulfato de halistanol A (7) isolado de Petromica ciocalyptoides apresentou atividade inibidora da enzima adenosina fosforribosil transferase isolada de Leishmania tarentolae de maneira dose-dependente, com inibição de $92 \%$ em uma concentração de $25 \mu \mathrm{g} / \mathrm{mL}$ ou $\mathrm{IC}_{50}$ de $2,87 \mu \mathrm{g} / \mathrm{mL}$. O composto 7 não apresentou atividade inibitória frente outras PRTases, tais como a hipoxantina-guaniafosforribosil-tranferase (HGPRT), xantinafosforribosil-tranferase (XPRT) e H-APRT (adenina-fosforribosiltransferase de Homo sapiens). Como é bem conhecido o fato de compostos sulfatados apresentarem diversas atividades biológicas devido à presença de seus grupos sulfato ${ }^{24}$, realizou-se a hidrólise destes grupos do trissulfato de halistanol $\left(\mathrm{H}_{2} \mathrm{SO}_{4} 10 \%\right.$, refluxo, $\left.3 \mathrm{~h}\right)$, de maneira a fornecer o correspondente esterol triidroxilado 8. O espectro de RMN${ }^{13} \mathrm{C}\left(\mathrm{MeOH}-d_{4}, 125 \mathrm{MHz}\right)$ de 8 indicou que ocorreu a dessulfatação dos grupos hidroxila, uma vez que o deslocamento químico dos carbonos oximetínicos antes observados em $\delta 75,7,75,8$ e 78,8 para o composto 7 foram subseqüentemente observados em $\delta$ 69.8, 69,0 e 67,5. O composto 8 obtido mostrou ser inativo como inibidor da LAPRT. De maneira a verificar se somente os grupos sulfato seriam responsáveis pela atividade observada, diferentes sais de sulfato de $\mathrm{Na}, \mathrm{K}$ e Mg foram submetidos ao bioensaio, não apresentando qualquer atividade. Sendo assim, apesar dos grupos sulfato do trissulfato de halistanol A serem essenciais para a atividade de inibição da LAPRT, devem apresentar uma posição espacial necessária para promover a inibição enzimática. O trissulfato de halistanol A foi subseqüentemente testado em promastigotas de Leishmania chagasi, mas não apresentou qualquer atividade antiparasitária.

Com o objetivo de se avaliar a atividade antifúngica do trissulfato de halistanol A em cepas de Candida spp. sensível (C. parapsilosis) e resistente (C. krusei) a antibióticos pertencentes à classe dos azóis, utilizou-se o ensaio de microdiluição em placas de 96 poços. Neste bioensaio o trissulfato de halistanol A foi mais efetivo contra $C$. krusei resistente aos azóis, apresentando uma $\mathrm{CI}_{50}$ de $25,81 \mu \mathrm{g} / \mathrm{mL}$ (intervalo de confiança 95\%: 16,61 - 40,10 $\mu \mathrm{g} / \mathrm{mL}$ ). A anfotericina B foi utilizada como fármaco padrão e apresentou uma $\mathrm{CI}_{50}$ de 49,46 ng/mL contra C. krusei e 18,52 ng/mL contra C. parapsilosis. A linhagem sensível $C$. parapsilosis apresentou-se menos suscetível à atividade antibiótica do trissulfato de halistanol A, com $\mathrm{CI}_{50}$ de 59,01 $\mu \mathrm{g} / \mathrm{mL}$ (intervalo de confiança $95 \%-57,53-60,52 \mu \mathrm{g} / \mathrm{mL}$ ). A atividade fungicida medida pelo método MTT indicou que o trissulfato de halistanol A promoveu inibição de $100 \%$ das leveduras na concentrações mais elevadas $(150 \mu \mathrm{g} / \mathrm{mL})$.

O mesmo trissulfato de halistanol A (7) foi isolado da esponja Topsentia ophiraphidites coletada na Baía de Todos os Santos (2004). Este foi caracterizado pela análise de seus espectros de RMN monoe bidimensionais, além de ter sido submetido ao procedimento de hidrólise dos grupos sulfato e acetilação de $\mathbf{8}$, fornecendo 9 com espectro de massas idêntico ao obtido a partir do mesmo composto 
isolado de $P$. ciocalyptoides. Este é o primeiro isolamento de um esterol sulfatado a partir de esponjas coletadas da costa do Brasil e o primeiro a apresentar inibição enzimática da L-APRT. Recentemente, dois triterpenos e um sesterterpeno dissulfatados foram isolados da esponja Callyspongia sp. oriunda de São Sebastião (SP) e também apresentaram atividade inibidora da L-APRT ${ }^{25}$.

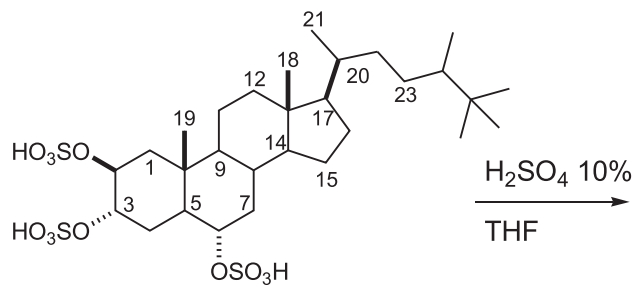

7<smiles>CC(CCC(C)C(C)(C)C)C1CCC2C3C[C@H](O)C4C[C@@H](O)C(O)CC4(C)C3CCC12C</smiles><smiles>CC(=O)OC1CC2(C)C3CCC4(C)C(C(C)CCC(C)C(C)(C)C)CCC4C3C[C@H](OC(C)=O)C2C[C@H]1OC(C)=O</smiles>

\section{Investigação química da ascídia Didemnum ligulum}

Ascídias são um grupo de invertebrados exclusivamente marinhos, tipicamente bioprodutores de compostos nitrogenados ${ }^{26}$. Dentre estes, destacam-se uma enorme variedade de peptídeos, alcalóides e derivados nitrogenados. Ascídias pertencentes ao gênero Didemnum (Filo Cordata, Classe Ascidiacea) são uma rica reserva de metabólitos marinhos estruturalmente diversificados e biologicamente ativos. A maioria dessas substâncias são compostos nitrogenados derivados de aminoácidos, como peptídeos cíclicos e acíclicos e alcalóides aromáticos ${ }^{26}$.

No presente estudo, o extrato metanólico da ascídia Didemnum ligulum oriunda de São Sebastião (SP) apresentou atividade antimicobacteriana contra Mycobacterium tuberculosis H37Rv. O extrato bruto foi fracionado por cromatografia de permeação em gel em coluna de Sephadex LH-20 (MeOH) e por cromatografia de fase reversa em coluna de sílica-gel derivatizada com 18 átomos de carbono $-\mathrm{C}_{18}$ (gradiente de $\mathrm{MeOH}$ em $\mathrm{H}_{2} \mathrm{O}$ ). A fração contendo compostos nitrogenados de caráter básico (resposta positiva frente ao reagente de Dragendorff em CCD) cristalizou e o sobrenadante foi purificado por cromatografia líquida de alta eficiência, utilizando-se uma coluna de sílica-gel derivatizada com grupos fenila. O composto cristalino foi identificado como sendo a asterubina (10) por análise por difração em raios-X (Tabelas 2 e 3), enquanto que o produto purificado por HPLC mostrou ser a $N, N$-dimetil- $O$-metiletanolamina (11), identificada pela análise de seus dados espectroscópicos. Posteriormente foram obtidos os dados de RMN ${ }^{1} \mathrm{H}, \mathrm{RMN}{ }^{13} \mathrm{C}$ e HMQC da asterubina (10), que possibilitaram a atribuição dos seus sinais de ${ }^{1} \mathrm{Hs}$ e ${ }^{13} \mathrm{Cs}$, ainda não relatada na literatura.

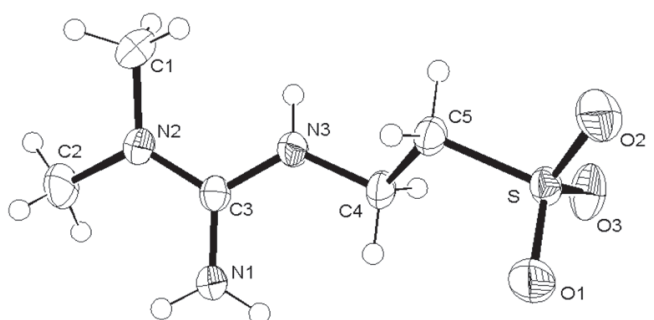

Figura 1. Representação ORTEP da molécula de asterubina (10) com os átomos identificados

Tabela 2. Dados cristalográficos da asterubina (10)

Fórmula empírica
Dimensões do cristal
Sistema cristalino
Grupo espacial
a $(\AA)$
b $(\AA)$
c $(\AA)$
$\beta\left({ }^{\circ}\right)$
V $\left(\AA^{3}\right)$
$Z$
F $(000)$
Densidade calculada
$\mu$ (mm $\left.{ }^{-1}\right)$
Difratômetro
Radiação, $\lambda(\AA)$
Temperatura $(\mathrm{K})$
Intervalo de $\theta$
Intervalo de hkl
Reflexões medidas
Reflexões únicas / Rint
Reflexões observadas $(\mathrm{I} \geq 2 \sigma(\mathrm{I}))$
Parâmetros refinados / restrições
$\mathrm{R}($ obs/all)
wR (obs/all)
$\mathrm{S}$
$\Delta \rho$ min/max (e $\left.\AA^{-3}\right)$

$\mathrm{C}_{5} \mathrm{H}_{3} \mathrm{~N}_{3} \mathrm{SO}_{3}$
$0,2 \times 0,2 \times 0,1$
Monoclínico
$\mathrm{P} 2 / \mathrm{c}$
$6,0852(5)$
$8,7508(8)$
$16,553(1)$
$97,063(6)$
$874,8(1)$
4
416
1,483
0,345
$\mathrm{CAD} 4$
$\mathrm{MoK} \alpha, 0,71073$
293
$2,48-29,95$
$-8 \leq \mathrm{h} \geq 0,0 \leq \mathrm{k} \geq 12$,
$-23 \leq 1 \geq 23$
2759
$2540 / 0,024$
1752
$120 / 0$
$0,043 / 0,086$
$0,107 / 0,122$
1,032
$-0,354 / 0,461$

Tabela 3. Geometria das ligações de hidrogênio da asterubina (10)

\begin{tabular}{lccccc}
\hline D-H & d(D-H) & d(H..A) & $<$ DHA & d(D..A) & A \\
\hline N1-HN1a & $0,84(2)$ & $2,10(2)$ & $163(2)$ & $2,917(2)$ & O3 $^{\text {i }}$ \\
N1-HN1b & $0,84(2)$ & $2,04(2)$ & $163(2)$ & $2,860(2)$ & O2 $^{\text {ii }}$ \\
N3-HN3 & $0,87(2)$ & $2,03(2)$ & $157(2)$ & $2,855(2)$ & O1 ${ }^{\text {iii }}$ \\
\hline
\end{tabular}

Operações de simetria: (i) $\mathrm{x},-\mathrm{y}+1 / 2, \mathrm{z}-1 / 2$, (ii) $-\mathrm{x}, \mathrm{y}-1 / 2,-\mathrm{z}+1 / 2$, (iii) $\mathrm{x}+1, \mathrm{y}, \mathrm{z}$

Isolada na forma de um sólido vítreo amarelo, a substância inédita $N, N$-dimetil- $O$-metiletanolamina (11) teve sua estrutura determinada pela análise de seus dados espectroscópicos (RMN ${ }^{1} \mathrm{H} \mathrm{e}{ }^{13} \mathrm{C}$, HMQC, HMBC, COSY $\left.{ }^{1} \mathrm{H}-{ }^{1} \mathrm{H}\right)$ e espectrometria de massas de alta resolução em modo $\mathrm{FAB}$, cujo espectro apresentou pico de íon pseudo-molecular $[\mathrm{M}+\mathrm{H}]^{+}$em $\mathrm{m} / z$, 104,10813 (calculado: 104,10754), compatível com a fórmula $\mathrm{C}_{5} \mathrm{H}_{14} \mathrm{NO}$. O grupo metoxila, com sinal no espectro de $\mathrm{RMN}-{ }^{1} \mathrm{H}\left(\mathrm{MeOH}-d_{4}, 100 \mathrm{MHz}\right)$ em $\delta 3,2$ $\left(\delta^{13} \mathrm{C} 54,7\right)$ mostrou estar acoplado ao grupo metileno em $\delta 3,5$ $\left(\delta^{13} \mathrm{C} 69\right)$ o qual, por sua vez, mostrou estar acoplado ao outro grupo metileno em $\delta 4,0\left(\delta^{13} \mathrm{C} 57,0\right)$, que constitui um sistema do tipo 
AA'BB' similar ao observado para o composto 6. O átomo de nitrogênio demonstrou estar substituído por dois grupos metila, que apresentaram sinal no espectro de $\mathrm{RMN}-{ }^{1} \mathrm{H}$ em $\delta 2,7\left(\delta^{13} \mathrm{C} 40,5\right)$. A asterubina já havia sido isolada anteriormente de uma estrela do mar (Asteria rubens) ${ }^{27}$ e mais recentemente da esponja Phoriospongia $s p .^{28}$, mas nunca de uma ascídia. Tal fato pode indicar que a ocorrência de compostos estruturalmente relacionados pode ser ampla, inclusive em organismos com pouca relação filogenética. A etanolamina é na verdade considerada um metabólito primário, sendo constituinte estrutural da colina e da acetilcolina, além de vários derivados lipídicos polares ${ }^{29}$. Tanto a asterubina (10) como a $\mathrm{N}, \mathrm{N}$-dimetil- $\mathrm{O}$-metiletanolamina (11) mostraram ser inativas em testes de atividades antimicrobiana e antimicobacteriana.

\section{Investigação química do octocoral Carijoa riisei}

Octocorais do gênero Carijoa, também conhecido como Telesto, foram objetos de diversas investigações químicas, tendo sido isolados prostanóides, amidas, esteróis glicosilados citotóxicos e eicosanóides halogenados com atividade antitumoral e anti-inflamatória ${ }^{30-35}$.

No presente estudo, o extrato $\mathrm{MeOH}$ do octocoral Carijoa riisei coletado em São Sebastião (SP) apresentou atividade citotóxica contra linhagens de células MCF-7 (câncer de mama), melanoma e cólon. Seu extrato bruto também apresentou atividade antimicrobiana contra Staphylococcus aureus, Pseudomonas aeruginosa, Enterobacter spp e Proteus spp, todas bactérias resistentes a antibióticos. $\mathrm{O}$ fracionamento desse extrato bruto por cromatografia rápida em coluna de sílica gel e por cromatografia líquida de alta eficiência (HPLC) resultou no isolamento de um único constituinte. A identificação deste composto foi realizada pela análise dos seus dados espectroscópicos $\left(\mathrm{RMN}-{ }^{1} \mathrm{H}\right.$ e ${ }^{13} \mathrm{C}$, HMQC, HMBC e COSY ${ }^{1} \mathrm{H}-{ }^{1} \mathrm{H}$ ), bem como por comparação com dados existentes na literatura, demonstrando tratar-se do esteróide 18-acetoxipregna-1,4,20-trien-3-ona (12), o primeiro metabólito secundário isolado deste octocoral ${ }^{35}$. Foi possível caracterizar agora completamente este composto, o que não foi feito quando de sua publicação original. A 18-acetoxipregna-1,4,20-trien-3-ona (12) isolada do octocoral Carijoa riisei na presente investigação apresentou moderada atividade em linhagens de células tumorais SF295 (sistema nervoso central) com $\mathrm{IC}_{50}$ de $14,4 \mu \mathrm{g} / \mathrm{mL}$, MDA-MB435 (mama) com $\mathrm{IC}_{50}$ de $23,1 \mu \mathrm{g} / \mathrm{mL}$, HCT8 (cólon) com $\mathrm{IC}_{50}$ de 22,0 $\mu \mathrm{g} / \mathrm{mL}$ e HL60 (leucemia) com $\mathrm{IC}_{50}$ de $12,4 \mu \mathrm{g} / \mathrm{mL}$.

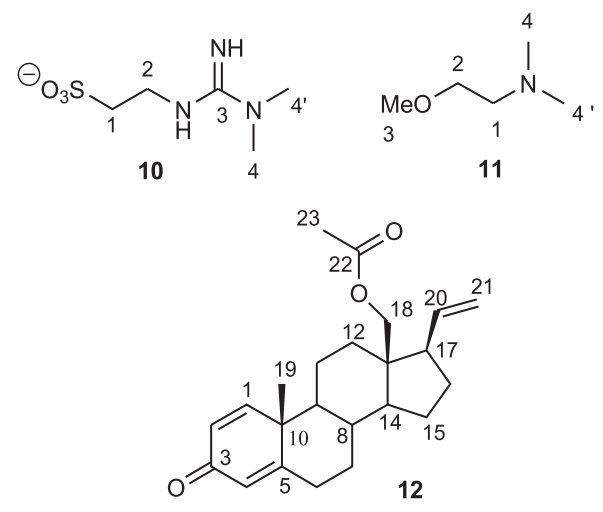

\section{CONCLUSÃO}

Neste trabalho são apresentados os resultados da investigação química dos extratos brutos de quatro espécies de esponjas, uma ascídia e um octocoral, levando ao isolamento de um composto inédito e 9 conhecidos. Dos 10 compostos isolados, 5 apresentaram atividades antimicrobianas. O alcalóide guanidínico isoptilocaulina, isolado de Monanchora arbuscula, mostrou ser um potente antibiótico contra linhagens de $S$. aureus resistentes à oxacilina, sendo este o primeiro estudo da atividade antibacteriana da isoptilocaulina contra linhagens resistentes de bactérias. De Aplysina sp. foram isolados 5 compostos derivados da dibromotirosina, dos quais 3 demonstraram ser inativos em testes de atividade antimicrobiana. O composto já conhecido trissulfato de halistanol A, isolado das esponjas Petromica ciocalyptoides e Topsentia ophiraphidites, apresentou atividade inibitória da enzima adenosina fosforibosil transferase isolada de Leishmania tarantolae, bem como atividade inibitória de Candida krusei resistente a antibióticos. A asterubina e a $N, N$-dimetil- $O$ metiletanolamina, ambas isoladas da ascídia Didemnum ligulum, mostraram ser inativas nos bioensaios de atividade antimicobacteriana. $\mathrm{O}$ esterol 18-acetoxipregna-1,4,20-trien-3-ona foi isolado a partir do octocoral Carijoa riisei e demonstrou apresentar moderada atividade citotóxica.

\section{PARTE EXPERIMENTAL}

\section{Generalidades}

Os espectros na região do infravermelho foram obtidos em um aparelho FT-IR Bomem MB102 em pastilha de sílica. Para as medidas de rotação específica foi usado o polarímetro 241 PerkinElmer. Os espectros na região do ultravioleta foram obtidos em $\mathrm{MeOH}$, em um aparelho Hitachi U-3210. Os dados de difração de raio-X foram coletados em um difratômetro automático CAD4 Enraf-Nonius, a $273 \mathrm{~K}$ no modo $\omega-2 \theta$. Os espectros de RMN foram obtidos em DMSO- $d_{6}, \mathrm{MeOH}-d_{4}, \mathrm{MeCN}-d_{3}$ ou $\mathrm{CDCl}_{3}$, a $25^{\circ} \mathrm{C}$ utilizando TMS como padrão interno, nos seguintes aparelhos: Bruker AC-4.7 Tesla, operando em 200,1 MHz (1H) e 50,3 MHz (13C); Bruker ARX 9.4 Tesla operando em 400,35 MHz $\left({ }^{1} \mathrm{H}\right)$ e $100,10 \mathrm{MHz}\left({ }^{13} \mathrm{C}\right)$ e Bruker DRX500 11,7 tesla operando em 500,13 $\mathrm{MHz}\left({ }^{1} \mathrm{H}\right)$ e $125,76 \mathrm{MHz}\left({ }^{13} \mathrm{C}\right)$ equipado com sonda multinuclear com gradiente de campo no eixo Z. Os espectros de massas foram obtidos utilizando dois equipamentos: espectrômetro de massas VG7070 operando a uma voltagem de aceleração nominal de $70 \mathrm{eV}$, no qual os espectros obtidos por EI, FAB ou CI foram calibrados com perfluoroquerosene, álcool 3-nitrobenzílico ou polietilenoglicol; equipamento Hybrid Kratos Concept IIHQ, em modo FAB. Solventes de grau analítico foram utilizados para a extração e cromatografia em coluna de baixa pressão "flash". Solventes de grau cromatográfico (HPLC) e água MilliQ foram utilizados em separações por cromatografia líquida, utilizando um equipamento Waters de bomba quaternária 600, detector de duplo feixe UV 2487 e processador-registrador 746. Vouchers das espécies de esponjas coletadas foram depositados na coleção de poríferos do Museu Nacional, Universidade Federal do Rio de Janeiro, Rio de Janeiro, RJ. Um voucher da ascídia Didemnum ligulum foi depositado na coleção de Ascidiacea do Departamento de Zoologia, Setor de Ciências Biológicas da Universidade Federal do Paraná. A gorgônia C. riisei foi identificada pelo Dr. A. E. Migotto (Centro de Biologia Marinha, Universidade de São Paulo).

\section{Ensaio da atividade antimicrobiana in vitro}

Para a determinação da CIM (Concentração Inibitória Mínima) foram utilizadas 8 cepas microbianas (7 bactérias e 1 levedura, mencionadas na discussão dos resultados e Tabela 1) e a técnica da microdiluição ${ }^{36}$. O inóculo foi preparado transferindo-se quatro 
a seis colônias de cada bactéria para caldo Mueller-Hinton, incubando-se por $6 \mathrm{~h}$ a $37^{\circ} \mathrm{C}$, de modo a se obter uma turbidez de $10^{6}$ bactérias/mL. Inóculo de $100 \mu \mathrm{L}$ de cada suspensão de células foi colocado nos orifícios da microplaca contendo $100 \mu \mathrm{L}$ de caldo Mueller-Hinton acrescido da substância em diferentes concentrações $(1250,625,312,250,170,85,42,5,21,10,5,2$ e $1 \mu \mathrm{g} / \mathrm{mL})$. A seguir, incubou-se a $37^{\circ} \mathrm{C}$ por $24 \mathrm{~h}$. O crescimento microbiano foi avaliado pela leitura da densidade óptica $(620 \mathrm{~nm}) \mathrm{em}$ aparelho ELISA (Multikan MS-Labsystem). Como controles negativos foram utilizados o caldo Mueller-Hinton esterilizado e culturas microbianas crescidas sem a adição das substâncias. Como controle positivo foram utilizados cloranfenicol, nistatina e cetoconazol. Todas as amostras foram testadas em duplicata. Nos ensaios onde se verificou a atividade sobre o crescimento da levedura Candida albicans, foi utilizado o meio de cultura GYW.

No bioensaio de atividade antifúngica, as leveduras Candida krusei (ATCC 6248) e Candida parapsilosis (ATCC 22019) foram mantidas em meio RPMI 1640, com L-glutamina (Gibco, Invitrogen) e glicose $2 \%$ em pH 7,0. O halistanol A foi dissolvido em DMSO e posteriomente diluído no meio RPMI 1640, utilizando-se microplacas de 96 poços. A suspensão do inóculo de leveduras foi ajustado para $1-5 \times 10^{5} \mathrm{cfu} / \mathrm{mL}$, sendo adicionados $100 \mu \mathrm{L}$ do inóculo da levedura em cada poço. A placa foi posteriomente incubada por $24 \mathrm{~h}$ à $35^{\circ} \mathrm{C}$ na presença do halistanol A. Após agitação, verificou-se o crescimento das leveduras em leitor de placas automático (Multiskan, Sweden) a $480 \mathrm{~nm}$. A anfotericina B (Fungizone) foi utilizada como controle positivo (antifúngico). A atividade fungicida ou fungistática do halistanol A foi avaliada através da viabilidade das leveduras, sendo acompanhada pelo método do $\mathrm{MTT}^{37}$. Os cálculos de concentração efetiva $50 \%$ foram realizados através de curvas sigmoidais dose-resposta e pelo software Graph Pad Prism 3.0, utilizando-se experimentos realizados em duplicata.

\section{Ensaios de inibição enzimática de adenosina fosforribosil transferase de Leishmania tarentolae}

Experimentos de cinética enzimática e inibição foram realizados em espectrofotômetro. A formação de mono-fosfato de adenosina (AMP) e mono-fosfato de guanosina (GMP) foi acompanhada espectrofotometricamente a 245 e $257,5 \mathrm{~nm}$, respectivamente, utilizando como coeficiente de extinção os valores de 3020 \pm 40 e $5340 \pm 50 \mathrm{M}^{-1} \mathrm{~cm}^{-1}$ respectivamente, em volume final de reação de $1 \mathrm{~mL}$.

Para a determinação da velocidade inicial de reação, adenina, hipoxantina ou guanina foram usados como substrato em concentração fixa contra diferentes concentrações do complexo de magnésio do pirofosfato de fosforribosila $\left(\mathrm{Mg}_{2} \mathrm{PRPP}\right)$ e vice-versa. Para a medição da velocidade inicial da reação reversa, tanto o complexo de magnésio do mono-fosfato de adenosina (MgAMP) como o complexo de magnésio do mono-fosfato de guanosina (MgGMP) foram usados como substrato em concentração fixa contra diferentes concentrações de pirofosfato inorgãnico de magnésio (MgPPi)ou inibidores e vice-versa.

\section{Teste de atividade antiparasitária contra Leishmania chagasi}

Hamsteres dourados (Mesocricetus auratus) foram fornecidos pelo biotério do Instituto Adolfo Lutz de São Paulo e mantidos em caixas esterilizadas com material absorvente, recebendo água e alimento ad libitum. Os hamsteres foram infectados a cada mês com amastigotas provenientes de baço para manutenção da cepa de Leishmania chagasi. Todos os procedimentos realizados nos ensaios foram previamente aprovados pela Comissão de Ética em Pesquisa do Instituto Adolfo
Lutz. Amastigotas de L. chagasi (MHOM/BR/1972/LD) foram purificados de baço de hamsteres dourados através de centrifugação diferencial, sendo a infecção avaliada pelo método de Stauber $^{36}$ em 60-70 dias após o inóculo. Promastigotas de $L$. chagasi foram cultivadas em meio M199 suplementado com $10 \%$ de soro fetal bovino, $0,25 \%$ de hemina e $5 \%$ de urina humana, a $24^{\circ} \mathrm{C}$. Com o objetivo de se verificar a efetividade do halistanol-A em $L$. chagasi, realizou-se a determinação da concentração efetiva $50 \%\left(\mathrm{CE}_{50}\right)$ em diferentes concentrações $(150 \mathrm{a} 0,073 \mu \mathrm{g} / \mathrm{mL})$, utilizando-se ainda o isotiocianato de pentamidina (Eurofarma) como fármaco padrão. O halistanol-A foi previamente dissolvido em DMSO e posteriormente diluído em meio M199, sendo incubado por um período de $48 \mathrm{~h}$ com $1 \times 10^{6}$ promastigotas/poço (previamente contados em hemocitômetro-Neubauer) em placas de 96 poços (triplicata). A viabilidade dos promastigotas foi verificada através de microscopia óptica e do ensaio com MTT ${ }^{37}$. Basicamente, o MTT (5 mg/mL) foi dissolvido em tampão fosfato-salina (PBS), esterilizado em membranas de $0,22 \mu \mathrm{m}$ e adicionado em um volume de 20 $\mu \mathrm{L} /$ poço. A placa foi novamente incubada por $4 \mathrm{~h}$ a $24{ }^{\circ} \mathrm{C}$. A extração da formazana foi realizada através da adição de dodecil sulfato de sódio (100 $\mu \mathrm{L} /$ poço) adicionando-se $10 \%$ em $\mathrm{HCl} 0,01 \mathrm{M}$ por um período de $18 \mathrm{~h}$. A densidade óptica dos poços foi verificada em um espectrofotômetro Multiskan MS no comprimento de onda de 570 $\mathrm{nm}$. Poços sem a adição de fármacos (formazana e dodecil sulfato de sódio) e com DMSO foram utilizados como controle de viabilidade do ensaio. A concentração efetiva 50\% (CE50) foi analisada pelo software Graph Pad Prism 3.0, através de uma curva sigmóide doseresposta, com base em pelo menos dois ensaios distintos.

\section{Ensaio da atividade citotóxica in vitro}

O método MTT foi utilizado para a determinação da atividade citotóxica de extratos e compostos puros. Colocou-se em microplacas de 96 orifícios, células aderentes de câncer em uma concentração de $2 \times 10^{3}$ células $/ 100 \mu \mathrm{L}$ e células suspensas (HL-60 e CEM) a uma concentração de $2 \times 10^{4}$ células $/ 100 \mu \mathrm{L}$. As células foram incubadas durante $24 \mathrm{~h}$. Os extratos foram adicionados em concentrações de $0,39-25 \mu \mathrm{g} / \mathrm{mL}$ às culturas e, em seguida, as células foram incubadas por 3 dias. Utilizou-se uma solução de MTT para finalizar o tempo de incubação. A sobrevivência das células foi avaliada em um espectrofotômetro de varredura multicanal a $540 \mathrm{~nm}$.

\section{Estudo químico da esponja Monanchora arbuscula}

A esponja Monanchora arbuscula foi coletada em seu habitat natural, na Baía de Todos os Santos, Salvador-BA e imediatamente congelada. Um espécimen padrão (MNRJ 2499) foi depositado no Museu Nacional, UFRJ. O material congelado foi liofilizado, extraído com $\mathrm{MeOH}$ (liqüidificador), filtrado e o extrato evaporado até obter-se uma suspensão aquosa, a qual foi diluída com $\mathrm{MeOH}$ de maneira a obter-se uma solução de composição aproximada $\mathrm{MeOH}-\mathrm{H}_{2} \mathrm{O}$ 9:1. Este extrato foi particionado com $n$-hexano. A fração $\mathrm{MeOH}-\mathrm{H}_{2} \mathrm{O}$ foi então evaporada, diluída em $\mathrm{H}_{2} \mathrm{O}$ e aplicada a uma resina XAD-2, a qual foi eluída primeiramente com $\mathrm{H}_{2} \mathrm{O}$ e depois com MeOH-AcOEt 1:1. A fração orgânica foi evaporada e o resíduo submetido a uma série de separações cromatográficas envolvendo cromatografias em colunas de sílica-gel derivatizada com grupos cianopropila, colunas de sílica-gel e, por fim, purificação por HPLC (fase reversa $\mathrm{C}_{18}$ ), de maneira a fornecer a isoptilocaulina (1) pura $(8,3 \mathrm{mg}, 0,005 \%)$.

\section{Isoptilocaulina (1)}

Goma marrom. HRFABMS: $m / z, 248,21197 ;[\alpha]_{D}^{25}=+21,2(\mathrm{c}$ 0,00335, MeOH); UV (MeOH) $\lambda_{\text {max }}(\varepsilon): 241$ (6025); IV (filme lí- 
quido): 3246, 2920, 2851, 1656, 1610, 1585, 1567, 1460, 1406, 1218, 1175, $1108 \mathrm{~cm}^{-1}$. RMN- ${ }^{1} \mathrm{H}\left(\mathrm{MeOH}-d_{4}, 400 \mathrm{MHz}\right): 3,89(\mathrm{~m}$, $1 \mathrm{H}, \mathrm{CH}-3 \mathrm{a}) ; 1,83$ (m, 2H, $\left.\mathrm{CH}_{2}-4\right) ; 1,96\left(\mathrm{~m}, 2 \mathrm{H}, \mathrm{CH}_{2}-5\right) ; 2,20$ (m, $1 \mathrm{H}, \mathrm{CH}-5 \mathrm{a})$; 1,37 (sl, 2H, CH-6); 4,01 (d, 5,4 Hz, 1H, CH-8a); 2,51 (m, 1H, CH-8b); 2,21 (m, 2H, $\mathrm{CH}_{2}-1^{\prime}$ ); 1,37 (sl, 2H, $\mathrm{CH}_{2}-2$ '); 1,37 (sl, 2H, $\mathrm{CH}_{2}-3$ ') 0,95 (t, 6,7 Hz; 3H, $\left.\mathrm{CH}_{3}-4^{\prime}\right) ; 1,78$ (s, 3H, $\left.\mathrm{CH}_{3}-9\right)$; $\mathrm{RMN}-{ }^{13} \mathrm{C}\left(\mathrm{MeOH}-d_{4}, 100 \mathrm{MHz}\right): 156,4(\mathrm{C}-2) ; 50,7(\mathrm{CH}-$ 3a); 35,3 ( $\left.\mathrm{CH}_{2}-4\right)$; 32,8 ( $\left.\mathrm{CH}_{2}-5\right)$; 37,5 (CH-5a); 31,9 ( $\left.\mathrm{CH}_{2}-6\right)$; 132,9 (C-7); 136,3 (C-8); 54,6 (CH-8a); 41,2 (CH-8b); 19,8 ( $\left.\mathrm{CH}_{3}-9\right)$; 37,1 $\left(\mathrm{CH}_{2}{ }^{\prime}{ }^{\prime}\right) ; 31,9\left(\mathrm{CH}_{2}-2^{\prime}\right) ; 23,8\left(\mathrm{CH}_{2}-3^{\prime}\right) ; 14,4\left(\mathrm{CH}_{3}-4^{\prime}\right)$.

\section{Estudo químico da esponja Aplysina sp.}

A esponja Aplysina sp., coletada na Baía de Todos os Santos, Salvador-BA (vouchers MNRJ 2578 e 4173 do Museu Nacional, UFRJ, Rio de Janeiro, RJ) e preservada em EtOH (696 g) foi filtrada e triturada em liquidificador com $\mathrm{MeOH}$. Ambos extratos $\mathrm{MeOH}$ e EtOH foram reunidos e evaporados até se obter uma suspensão aquosa. Em seguida, realizou-se uma partição da fase aquosa com AcOEt. O extrato em AcOEt foi submetido a uma cromatografia em coluna de sílica gel Sep-Pak (10 g) com um gradiente de $\mathrm{MeOH}$ em $\mathrm{CH}_{2} \mathrm{Cl}_{2}$. Foram obtidas 6 frações desta separação. A primeira fração obtida, AcEA-1, foi submetida a uma cromatografia em Sephadex LH-20 (MeOH). Desta separação foram obtidas 7 frações. Destas frações, a segunda (AcEA-1-2) foi submetida a uma cromatografia em coluna de sílica gel Sep-Pak ( $2 \mathrm{~g})$ com gradiente de $\mathrm{MeOH} / \mathrm{AcOEt}$ 1:1 em $\mathrm{CH}_{2} \mathrm{Cl}_{2}$. Das frações obtidas, a fração AcEA-1-2-2 mostrou ser constituída pela (3',5'-dibromo-4'-etoxi$1^{\prime}$-hidroxi-4'-metoxicicloexa-2',5'-dienila)acetamida (2) $(245,7$ $\mathrm{mg}, 0,04 \%$ ). A terceira fração (AcEA-1-3) foi submetida a uma cromatografia em coluna de sílica gel Sep-Pak (2 g) e purificada por HPLC, utilizando-se uma coluna Waters $\mu$ Bondapak Phenyl (7,8 x $300 \mathrm{~mm}$ ) com uma mistura de $\mathrm{MeOH} / \mathrm{H}_{2} \mathrm{O} 1: 1$ obtendo-se a aeroplisinina-2 (3) (1 mg, 0,0001\%) e o 7,9-dibromo-6-hidroxi-8metoxi-4-oxa-3-azaspiro[4.5]dec-2,7,9-triene-2-carboxilato de etila (4) $(1,4 \mathrm{mg}, 0,0002 \%)$. À fração AcEA-1-5 foi adicionado $\mathrm{MeOH}$ a frio, o que resultou na precipitação da [3,5-dibromo-4-[(2-oxo-5oxazolidinila)]metoxifenil]-2-oxazolidinona (5) (55 mg, 0,008\%). A fração AcEA-2 foi submetida a uma cromatografia em Sephadex LH-20 (MeOH), resultando em 8 frações. A segunda (AcEA-2-2) e a quinta (AcEA-2-5) frações desta separação foram purificadas por HPLC, em coluna Waters $\mu$ Bondapak Phenyl $(7,8$ x $300 \mathrm{~mm})$ com uma mistura de $\mathrm{MeOH} / \mathrm{H}_{2} \mathrm{O}$ 1:1 e em coluna CSC Inertsil 150A/ ODS $25 \mu \mathrm{m}(25 \times 0,94 \mathrm{~cm})$ com uma mistura de $\mathrm{ACN} / \mathrm{H}_{2} \mathrm{O}$ 65:35, respectivamente, que resultou no isolamento do 2-(3,5-dibromo-4metoxifenil)- $N, N, N$-trimetiletanamônio (6) $(7,9 \mathrm{mg}, 0,001 \%) \mathrm{e}$ novamente na substância (3) $(2,9 \mathrm{mg}, 0,0004 \%)$.

\section{(3',5'-dibromo-4'-etoxi-1 '-hidroxi-4'-metoxicicloexa-2',5'-} dienila)acetamida (2)

Sólido vítreo. $[\alpha]_{\mathrm{D}}^{25}=+1,80\left(\mathrm{c} 0,0167, \mathrm{CHCl}_{3}\right) ; \mathrm{UV}(\mathrm{MeOH})$ $\lambda_{\text {max }}$ (ع): $260 \mathrm{~nm}$ (6500); IV (filme líquido): 3415, 3351, 2978, 2896, $1669,1628,1440,1400,1322,1061 \mathrm{~cm}^{-1} . \mathrm{RMN}-{ }^{1} \mathrm{H}\left(\mathrm{CDCl}_{3}, 400\right.$ MHz): 6,75 (s, 2H, CH-2 e 6); 2,54 (s, 2H, $\mathrm{CH}_{2}-7$ ); 3,13 (s, 3H, $\mathrm{CH}_{3}-9$ ); 3,35 (q, 7,1 Hz, 2H, $\left.\mathrm{CH}_{2}-10\right) ; 1,20$ (t, 7,1 Hz, 3H, $\mathrm{CH}_{3}-$ 11);. $\mathrm{RMN}-{ }^{13} \mathrm{C}\left(\mathrm{CDCl}_{3}, 100 \mathrm{MHz}\right)$ : 96,7 (C-4); 124,1 (C-3 e 5); 140,3 (CH-2 e 6); 71,3 (C-1); 44,2 ( $\left.\mathrm{CH}_{2}-7\right)$; 173,2 (C-8); 51,3 ( $\mathrm{CH}_{3}-$ 9); 60,2 ( $\left.\mathrm{CH}_{2}-10\right) ; 15,4\left(\mathrm{CH}_{3}-11\right)$.

\section{Aeroplisinina-2 (3)}

Sólido vítreo. LREI: $m / z$ 339,8; $[\alpha]_{\mathrm{D}}^{25}=+57,1$ (c 0,0007,

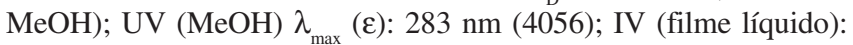
$3422,1774,1320,1200,1084 \mathrm{~cm}^{-1}$; RMN- ${ }^{1} \mathrm{H}\left(\mathrm{MeCN}-d_{3}, 500 \mathrm{MHz}\right)$ :
5,15 (s, 1H, CH-1); 6,46 (s, 1H, CH-5); 2,89 e 2,84 (d, 17,7 Hz, $\left.1 \mathrm{H}, \mathrm{CH}_{2}-7\right) ; 3,74$ (s, 3H, $\left.\mathrm{CH}_{3}-9\right)$; $\mathrm{RMN}^{-13} \mathrm{C}\left(\mathrm{MeCN}-d_{3}, 125 \mathrm{MHz}\right)$ : 89,1 (CH-1); 77,0 (C-2); 107,6 (C-3); 151,2 (C-4); 119,3 (C-5); 135,5 (CH-6); 42,2 ( $\left.\mathrm{CH}_{2}-7\right) ; 173,7$ (C-8); 61,5 ( $\left.\mathrm{CH}_{3}-9\right)$.

\section{2,4-dibromo-1-hidroxi-3-metoxi-6-oxa-3-azaspiro[4.5]dec-2,4,8- trieno-8-carboxilato de etila (4) \\ Sólido vítreo. $[\alpha]_{\mathrm{D}}^{25}=+137,9(\mathrm{c} 0,0014, \mathrm{MeOH}) ; \mathrm{UV}(\mathrm{MeOH})$} $\lambda_{\max }(\varepsilon): 292 \mathrm{~nm}$ (2085); IV (filme líquido): 3422, 1719, 1595, 1262, $1127,990 \mathrm{~cm}^{-1}$; RMN- ${ }^{1} \mathrm{H}\left(\mathrm{ACN}-d_{3}, 500 \mathrm{MHz}\right): 4,16(\mathrm{~d}, 7,3 \mathrm{~Hz}, 1 \mathrm{H}$, $\mathrm{CH}-1)$; 6,41 (s, 1H, CH-5); 3,69 e 3,06 (d, 18,2 Hz; 1H, $\left.\mathrm{CH}_{2}-7\right)$; 4,29 (q, 7,1 Hz, 2H, CH -10 ); 1,30 (t, 7,1 Hz, 3H, $\mathrm{CH}_{3}-11$ ); 3,70 (s, $\left.3 \mathrm{H}, \mathrm{CH}_{3}-12\right)$; $\mathrm{RMN}-{ }^{13} \mathrm{C}$ (ACN- $\left.d_{3}, 125 \mathrm{MHz}\right): 75,1$ (CH-1); 113,8 (C-2); 149,0 (C-3); 122,3 (C-4); 132,3 (CH-5); 92,6 (C-6); 40,0 $\left(\mathrm{CH}_{2}-7\right) ; 153,3$ (C-8); 161,0 (C-9); 63,0 ( $\left.\mathrm{CH}_{2}-10\right) ; 14,4\left(\mathrm{CH}_{3}-11\right)$; $60,8\left(\mathrm{CH}_{3}-12\right)$.

\section{Estudo da esponja Petromica ciocalyptoides}

A esponja foi coletada na Baía de Todos os Santos em 1999 e imediatamente congelada. O material congelado foi liofilizado, extraído com $\mathrm{MeOH}$ (liqüidificador), filtrado e o extrato evaporado até obter-se uma suspensão aquosa, a qual foi diluída com $\mathrm{MeOH}$ de maneira a obter-se uma solução de composição aproximada $\mathrm{MeOH}-\mathrm{H}_{2} \mathrm{O}$ 9:1. Este extrato foi particionado com $n$-hexano. A fração polar foi evaporada, dissolvida em $\mathrm{MeOH}$ e submetida a uma separação por cromatografia de permeação em gel de Sephadex LH-20 (MeOH). A segunda fração obtida dessa cromatografia demonstrou ser ativa no bioensaio de inibição da L-APRT e foi purificada em uma coluna de fase reversa $\mathrm{C}_{18}$ eluída com um gradiente de $\mathrm{MeOH}$ em $\mathrm{H}_{2} \mathrm{O}$. A quarta fração obtida desta separação demonstrou ser ativa contra a L-APRT fornecendo o trissulfato de halistanol A puro $(0,154 \mathrm{~g}, 0,18 \%)$.

\section{Trissulfato de halistanol A (7)}

Sólido amorfo bege. $[\alpha]_{\mathrm{D}}^{29,4}=+9,97(\mathrm{c} 0,084, \mathrm{MeOH}) ; \mathrm{RMN}{ }^{1} \mathrm{H}$ (MeOH- $d_{4}, 400 \mathrm{MHz}$ ): 2,11 (dl, 14,3; $\left.\mathrm{CH}_{2}-1\right) ; 1,46$ (m; $\left.\mathrm{CH}_{2}-1\right) ; 4,75$ (sl; CH-2); 4,80 (sl; CH-3); 2,29 (dl, 14,5; $\left.\mathrm{CH}_{2}-4\right) ; 1,78$ (tl, 14,4; $\mathrm{CH}_{2}-4$ ); 1,62 (m; CH-5); 4,19 (dt, 4,4 e 10,9; CH-6); 2,36 (dl, 10,2; $\left.\mathrm{CH}_{2}-7\right) ; 1,04$ (m; $\left.\mathrm{CH}_{2}-7\right) ; 1,53$ (m; CH-8); 0,73 (m; CH-9); 1,33 (m; $\left.\mathrm{CH}_{2}-11\right) ; 2,01$ (dl, 12,4; $\left.\mathrm{CH}_{2}-12\right) ; 1,15$ (m; $\left.\mathrm{CH}_{2}-12\right) ; 1,16$ (m; CH14); 1,61 (m; $\left.\mathrm{CH}_{2}-15\right) ; 1,11$ (d, 6,3; $\left.\mathrm{CH}_{2}-15\right) ; 1,86\left(\mathrm{~m} ; \mathrm{CH}_{2}-16\right) ; 1,64$ (m; $\left.\mathrm{CH}_{2}-16\right) ; 1,13$ (m; CH-17); 0,69 (sl; $\left.\mathrm{CH}_{3}-18\right) ; 0,83$ (sl; $\left.\mathrm{CH}_{3}-19\right)$; 1,38 (m; CH-20); 0,95 (d, 7,4; $\left.\mathrm{CH}_{3}-21\right) ; 1,58$ (m; $\left.\mathrm{CH}_{2}-22\right) ; 0,90$ (m; $\left.\mathrm{CH}_{2}-22\right) ; 0,86$ (m; $\left.\mathrm{CH}_{2}-23\right) ; 0,99$ (m; $\left.\mathrm{CH}_{2}-24\right) ; 0,85$ (s; $\left.\mathrm{CH}_{3}-26\right)$; 0,85 (s; $\left.\mathrm{CH}_{3}-27\right) ; 0,83$ (d; $\left.\mathrm{CH}_{3}-28\right) ; 0,85$ (s; $\left.\mathrm{CH}_{3}-29\right) ; \mathrm{RMN}^{13} \mathrm{C}$ (MeOH- $\left.d_{4}, 100 \mathrm{MHz}\right): 39,2\left(\mathrm{CH}_{2}-1\right)$; 75,7 (CH-2); 75,8 (CH-3); 25,1 $\left(\mathrm{CH}_{2}-4\right) ; 45,4$ (CH-5); 78,8 (CH-6); 40,1 ( $\left.\mathrm{CH}_{2}-7\right) ; 35,2$ (CH-8); 55,8 (CH-9); 37,7 (C-10); 21,9 ( $\left.\mathrm{CH}_{2}-11\right) ; 41,3\left(\mathrm{CH}_{2}-12\right) ; 43,8$ (C-13); 57,6 (CH-14); 25,2 ( $\left.\mathrm{CH}_{2}-15\right) ; 29,2\left(\mathrm{CH}_{2}-16\right) ; 57,7$ (CH-17); 12,5 $\left(\mathrm{CH}_{3}-18\right) ; 15,3\left(\mathrm{CH}_{3}-19\right) ; 37,7(\mathrm{CH}-20) ; 19,6\left(\mathrm{CH}_{3}-21\right) ; 37,7\left(\mathrm{CH}_{2}-\right.$ 22); 22,03 ( $\left.\mathrm{CH}_{2}-23\right) ; 45,5\left(\mathrm{CH}_{2}-24\right) ; 34,1(\mathrm{CH}-25) ; 27,8\left(\mathrm{CH}_{3}-26\right)$; $27,8\left(\mathrm{CH}_{3}-27\right) ; 15,0\left(\mathrm{CH}_{3}-28\right) ; 27,8\left(\mathrm{CH}_{3}-29\right)$.

\section{Hidrólise do trissulfato de halistanol A (7)}

$\mathrm{O}$ trissulfato de halistanol A $(7,10 \mathrm{mg})$ foi refluxado em $1 \mathrm{~mL}$ de dioxano e $1 \mathrm{~mL}$ de uma solução de $\mathrm{H}_{2} \mathrm{SO}_{4} 10 \%$ em $\mathrm{H}_{2} \mathrm{O}$ durante $3 \mathrm{~h}^{38}$. Depois deste período, o meio reacional foi neutralizado com solução saturada de $\mathrm{NaHCO}_{3}$ e evaporado até a secura. O material obtido foi redissolvido em $\mathrm{H}_{2} \mathrm{O}$ e submetido à extração em fase sólida em coluna de sílica-gel derivatizada com $\mathrm{C}_{18}$ (Waters SepPak ${ }^{\circledR}$ $2 \mathrm{~g}$ ), eluída com $\mathrm{H}_{2} \mathrm{O}, \mathrm{H}_{2} \mathrm{O} / \mathrm{MeOH}$ 1:1 e $\mathrm{MeOH} 100 \%$. As frações obtidas foram evaporadas e analisadas por CCD (revelador: solu- 
ção de ácido fosfomolíbdico em EtOH, seguido de aquecimento em placa a $110{ }^{\circ} \mathrm{C}$ durante $2 \mathrm{~min}$ ). A fração eluída em $\mathrm{H}_{2} \mathrm{O} / \mathrm{MeOH}$ 1:1 apresentou o esterol dessulfatado $(6,7 \mathrm{mg}, 67 \%$ de rendimento), com o qual foram realizadas análises de $\mathrm{RMN}-{ }^{13} \mathrm{C}$ e $\mathrm{EM}$. HRESIMS [M - 2. $\left.\mathrm{H}_{2} \mathrm{O}+\mathrm{H}\right]^{+} \mathrm{m} / \mathrm{z}$ 413.2675, calc 412.37052. RMN ${ }^{13} \mathrm{C}$ (DMSO-d6, $75 \mathrm{MHz}$ ): 69.8, 69.0, 67.5, 56.1, 55.6, 54.5, 45.5, 43.6, 42.3, 42.1, 36.1, 35.1, 33.5, 33.1, 27.9, 27.7, 27.3, 25.3, 24.0, $20.5,19.1,15.3,14.7,12.1$.

Acetilação do trissulfato de halistanol A dessulfatado (8)

O composto $8(6,7 \mathrm{mg})$ foi transferido para um reator de $2 \mathrm{~mL}$ com $\mathrm{MeOH}$, o qual foi evaporado. A amostra foi dissolvida em 0,5 $\mathrm{mL}$ de piridina e, em seguida, adicionou-se $0,5 \mathrm{~mL}$ de anidrido acético. A reação foi mantida sob agitação magnética por uma noite. Após a reação, os reagentes foram evaporados e o produto purificado por cromatografia em coluna de sílica gel (Waters Sep Pak ${ }^{\circledR}, 2$ g) com um gradiente de $\mathrm{B}$ em $\mathrm{A}$, onde $\mathrm{A}$ foi hexano e $\mathrm{B}$ foi uma mistura de $\mathrm{CH}_{2} \mathrm{Cl}_{2}$-AcOEt 9:2, fornecendo 3,0 mg $(44,7 \%)$ do produto 9 .

\section{Estudo da esponja Topsentia ophiraphidites}

A esponja foi coletada na Baía de Todos os Santos em 2004 e imediatamente congelada. O material congelado (574,7 g) foi liofilizado, extraído com $\mathrm{MeOH}$ (liqüidificador), filtrado e o extrato evaporado até obter-se uma suspensão aquosa, a qual foi diluída com $\mathrm{MeOH}$ de maneira a obter-se uma solução de composição aproximada $\mathrm{MeOH}-\mathrm{H}_{2} \mathrm{O}$ 9:1. Este extrato foi particionado com $n$ hexano. A fração hidroalcoólica foi evaporada e ressuspendida em $\mathrm{H}_{2} \mathrm{O}$, sendo em seguida submetida à outra partição com AcOEt (300 mL, repetindo-se por três vezes), obtendo-se duas frações: a fração AcOEt e a aquosa. A fração aquosa foi evaporada, dissolvida em $\mathrm{MeOH}$ e submetida a uma separação cromatográfica em coluna de Sephadex LH-20 (MeOH). A segunda fração obtida dessa cromatografia demonstrou ser ativa no bioensaio de inibição da enzima L-APRT e foi purificada em uma coluna de fase reversa $\mathrm{C}_{18}$ eluída com um gradiente de $\mathrm{MeOH}$ em $\mathrm{H}_{2} \mathrm{O}$. A quinta fração desta separação demonstrou ser ativa contra a L-APRT, fornecendo o trissulfato de halistanol A puro, o mesmo composto isolado da esponja Petromica ciocalyptoides (22,0 mg, 0,003\%).

\section{Estudo químico da ascídia Didemnum ligulum}

A ascídia (204 g) foi coletada em São Sebastião-SP em 1999 e conservada em EtOH. O material foi extraído exaustivamente com $\mathrm{MeOH}$, filtrado e evaporado. O extrato evaporado foi diluído com $\mathrm{MeOH} / \mathrm{H}_{2} \mathrm{O}$ 9:1 e particionado com $n$-hexano. A fração polar foi evaporada, suspendida em $\mathrm{H}_{2} \mathrm{O}$ e submetida a uma partição líquido-líquido com AcOEt. O extrato AcOEt foi fracionado através de cromatografia de permeação em gel Sephadex LH-20 com eluição isocrática $(\mathrm{MeOH})$ e, em seguida, por cromatografia de fase reversa em coluna de sílicagel derivatizada com grupos octadecil $\left(\mathrm{C}_{18}\right)$ (Waters Sep Pak® $10 \mathrm{~g}$ ) utilizando-se gradiente $\mathrm{MeOH}$ em $\mathrm{H}_{2} \mathrm{O}$. A fração contendo compostos nitrogenados (positiva ao reagente de Dragendorff) foi dissolvida em $\mathrm{MeOH}-d_{4}(500 \mu \mathrm{L})$, transferida para um tubo de RMN e mantida em geladeira a $4{ }^{\circ} \mathrm{C}$. Após $24 \mathrm{~h}$ observou-se a formação de cristais monoclínicos, os quais foram cuidadosamente retirados do tubo e analisados por difração por raios-X. Posteriormente, os cristais foram dissolvidos em DMSO- $d_{6}$ e analisados por $\mathrm{RMN}-{ }^{1} \mathrm{H}, \mathrm{RMN}-{ }^{13} \mathrm{C}, \mathrm{COSY}$ ${ }^{1} \mathrm{H}-{ }^{1} \mathrm{H}$ e HMQC. O composto cristalino foi identificado como sendo a asterubina (10) $(4,0 \mathrm{mg}, 0,002 \%)$. O sobrenadante em $\mathrm{MeOH}-d_{4}$ foi purificado por cromatografia líquida em coluna de fase reversa, utilizando-se uma coluna Waters $\mu$ Bondapak $^{\text {TM }}$ fenil $(125 \AA / 10 \mu \mathrm{m} 7,8 \mathrm{x}$ $300 \mathrm{~mm}$ ), com uma mistura de $\mathrm{MeOH} / \mathrm{H}_{2} \mathrm{O}$ (70:30) como eluente. A separação foi monitorada em $212 \mathrm{~nm}$, obtendo-se a $N, N$-dimetil- $O$ metiletanolamina (11) $(10,4 \mathrm{mg}, 0,005 \%)$.

\section{Asterubina (10)}

Sólido cristalino incolor, HRESIMS: $\mathrm{m} / \mathrm{z}, 218,0578,[\mathrm{M}+\mathrm{Na}]^{+}$; RMN ${ }^{1} \mathrm{H}$ (DMSO- $d$, $400 \mathrm{MHz}$ ): 7,53 (s, 3NH); 3,44 (dd, 4,7 e 11,7 $\left.\mathrm{Hz} ; 2 \mathrm{H}, \mathrm{CH}_{2}-2\right)$; 2,94 (s, 6H, $\left(\mathrm{CH}_{3}\right)_{2}-4$ e 4'); 2,72 (t, 4,7 Hz; $2 \mathrm{H}$, $\mathrm{CH}_{2}-1$ ); $\mathrm{RMN}{ }^{13} \mathrm{C}$ (DMSO-d, $100 \mathrm{MHz}$ ): 155,7 (C-3); 39,0 (C-2); 49,1 (C-1) e 37,8 (C-4 e 4').

\section{$N, N$-dimetil-O-metiletanolamina (11)}

Sólido vítreo amarelo, HRFABMS: $\mathrm{m} / \mathrm{z}$ 104,10813; IV (filme líquido): 3369, 1668, 1622, $1202 \mathrm{~cm}^{-1}$ : RMN- ${ }^{1} \mathrm{H}\left(\mathrm{MeOH}-d_{4}, 400\right.$ $\mathrm{MHz})$ : 4,0 (m, 2H, $\left.\mathrm{CH}_{2}-1\right) ; 3,5\left(\mathrm{~m}, 2 \mathrm{H}, \mathrm{CH}_{2}-2\right)$; 3,2 (s, 3H, $\left.\mathrm{CH}_{3}-3\right)$ e 2,7 (s, $6 \mathrm{H}, \mathrm{CH}_{3}-4$ e $\left.4^{\prime}\right)$; $\mathrm{RMN}-{ }^{13} \mathrm{C}\left(\mathrm{MeOH}-d_{4}, 100 \mathrm{MHz}\right) ; 57(\mathrm{C}-$ 1); 69 (C-2); 54,7 (C-3) e 40,5 (C-4 e 4').

\section{Determinação da estrutura cristalina da asterubina (10)}

Os dados de difração da asterubina (10) foram coletados em um difratômetro automático CAD4 Enraf-Nonius, a $273 \mathrm{~K}$ no modo $\omega$ 20. Os parâmetros de cela unitária foram obtidos e refinados usando 22 reflexões centradas automaticamente. Na Tabela 2 estão listados os principais dados cristalográficos e as condições de refinamento. Os dados de intensidade foram corrigidos pelos fatores de absorção usando o Método PSISCAN ${ }^{39}$. A estrutura foi resolvida através do $\mathrm{SIR}^{4} 2^{40}$ e refinada pelo método de mínimos quadrados com matriz completa e sucessivas sínteses de Fourier diferença, usando o sistema WinGX ${ }^{41}$ Os átomos de hidrogênio ligados aos átomos de $\mathrm{N}$ foram localizados em mapas de Fourier diferença e foram incluídos no refinamento com parâmetros de deslocamento térmico 1,2 vezes o Ueq do átomo ao qual se ligam. Os demais átomos de hidrogênio foram colocados em suas posições ideais com parâmetros de deslocamento térmico 1,5 vezes o Ueq do átomo ao qual se ligam para hidrogênios de metilas e 1,2 para os demais. Todos os átomos não hidrogênio foram refinados anisotropicamente. Uma representação ORTEP $^{42}$ da molécula com os átomos identificados é apresentada na Figura 1. No empacotamento cristalino a molécula de asterubina forma três ligações de hidrogênio intermoleculares entre N1 e O3, N1 e $\mathrm{O} 2$ e N3 e O1. A geometria das ligações de hidrogênio, com respectivas operações de simetria, é apresentada na Tabela 3.

\section{Estudo do octocoral Carijoa riisei}

O animal (89 g) foi coletado em São Sebastião (SP) em 1997 e imediatamente imerso em EtOH e mantido a $-20{ }^{\circ} \mathrm{C}$. O octocoral foi separado do $\mathrm{EtOH}$ de estocagem e triturado em $\mathrm{MeOH}$. Ambos extratos $\mathrm{EtOH}$ e $\mathrm{MeOH}$ foram reunidos, filtrados e evaporados até um volume de aproximadamente $300 \mathrm{~mL}$. A suspensão hidroalcóolica foi particionada com $n$-hexano, resultando em duas frações que foram evaporadas, pesadas e avaliadas por CCD utilizando-se como reveladores lâmpada de UV ( $\lambda=254 \mathrm{~nm})$, Dragendorff, ácido fosfomolíbdico e ninidrina. A fração hexânica foi submetida a uma cromatografia rápida em coluna de sílica gel 230-400 Mesh, utilizando-se um gradiente de AcOEt em $n$-hexano. A quarta fração resultante desta cromatografia foi purificada por cromatografia líquida de alta eficiência (HPLC) utilizando-se uma coluna de fase reversa $\mathrm{C}_{18}$ CSC-inertsil (150 ̊̊/ODS2, $5 \mu \mathrm{m}$ ) com uma mistura de $\mathrm{H}_{2} \mathrm{O} /$ acetonitrila/isopropanol (35:15:50) como eluente e monitorada em $280 \mathrm{~nm}$. Obteve-se assim, a 18-acetoxipregna1,4,20-trien-3-ona (12) (5 mg, 0,006\%).

18-acetoxipregna-1,4,20-trien-3-ona (12)

Sólido vítreo amarelo, LRESIMS: m/z $377[\mathrm{M}+\mathrm{Na}]^{+}$; UV $(\mathrm{MeOH}) \lambda_{\text {máx }}(\varepsilon): 245(15600) ;[\alpha]_{\mathrm{D}}^{25}=+36,25(\mathrm{c} 0,0032, \mathrm{MeOH})$; 
IV (filme líquido): 2939, 1740, 1737, 1663, 1239, 1030, $900 \mathrm{~cm}^{-1}$. RMN-1 H (DMSO- $d_{6}, 500$ MHz): 7,2 (d, 10,1 Hz; CH-1); 6,11 (dd, 10,1 e 1,85 Hz; CH-2); 6,0 (s; CH-4); 2,50 (ddd, 16,0, 5,0 e 2,0 Hz; $\mathrm{CH}_{2}-6$ ); 2,33 (dt, 12,8 Hz; $\mathrm{CH}_{2}-6$ ); 0,96 (dd, 13,0 e 2,0 Hz; $\mathrm{CH}_{2}-7$ ); 1,95 (d; 13,0 Hz; $\left.\mathrm{CH}_{2}-7\right) ; 0,73$ (m; CH-8); 1,04 (m; CH-9); 1,24 (m; $\mathrm{CH}_{2}-11$ ); 1,69 (m; $\mathrm{CH}_{2}-11$ ); 1,55 (ddd, 26,0, 13,0 e 3,5 Hz; $\mathrm{CH}_{2}-12$ ); 1,71 (m; $\left.\mathrm{CH}_{2}-12\right) ; 1,22$ (m, 12,4; $\left.\mathrm{CH}-14\right) ; 1,80$ (m; $\left.\mathrm{CH}_{2}-15\right) ; 1,68$ (m; $\left.\mathrm{CH}_{2}-15\right) ; 2,05$ (m; $\left.\mathrm{CH}_{2}-16\right) ; 1,05$ (m; $\left.\mathrm{CH}_{2}-16\right) ; 2,08$ (m; CH-17); 4,05 (d, $\left.12 \mathrm{~Hz} ; \mathrm{CH}_{2}-18\right) ; 3,98$ (d, 11,8 Hz; $\left.\mathrm{CH}_{2}-18\right) ; 1,20$ (sl; $\left.\mathrm{CH}_{3}-19\right) ; 5,79$ (ddd, 17,25, 10,35 e 7,6 Hz; CH-20); 4,94 (d, 10,4 Hz; $\mathrm{CH}_{2}-21$ ); 4,90 (d, $1,96 \mathrm{~Hz} ; \mathrm{CH}_{2}-21$ ); 2,0 (s; $\left.\mathrm{CH}_{3}-23\right)$; $\mathrm{RMN}-{ }^{13} \mathrm{C}$ (DMSO- $\left.d_{6}, 125 \mathrm{MHz}\right)$ : 156,6 (CH-1); 127,0 (CH-2); 185,2 (C-3); 123,3 (CH-4); 169,5 (C-5); 32,1 ( $\left.\mathrm{CH}_{2}-6\right) ; 33,6\left(\mathrm{CH}_{2}-7\right) ; 35,0(\mathrm{CH}-8) ; 52,3$ (CH-9); 43,4 (C-10); $24,5\left(\mathrm{CH}_{2}-11\right) ; 22,2\left(\mathrm{CH}_{2}-12\right) ; 46,2(\mathrm{C}-13) ; 53,7(\mathrm{CH}-14) ; 26,8\left(\mathrm{CH}_{2}-\right.$ 15); 32,2 ( $\left.\mathrm{CH}_{2}-16\right) ; 54,1$ (CH-17); 61,5 ( $\left.\mathrm{CH}_{2}-18\right) ; 18,7\left(\mathrm{CH}_{3}-19\right) ; 139,32$ (CH-20); 114,13 (CH-21); 170,5 (C-22); 21,0 ( $\left.\mathrm{CH}_{3}-23\right)$.

\section{AGRADECIMENTOS}

Os autores agradecem a gentileza do Prof. B. Copp (University of Auckland, Auckland, Nova Zelândia), do Dr. D. E. Williams e Prof. R. J. Andersen (University of British Columbia, Vancouver, Canadá) pela ajuda na obtenção dos espectros de massas de baixa e alta resolução. Os autores também são gratos aos Profs. M. da G. B. S. Moreira, J. C. Freitas e E. Trajano, diretores do Centro de Biologia Marinha da Universidade de São Paulo (CEBIMar-USP), pelas muitas facilidades oferecidas durante as coletas de invertebrados da região de São Sebastião. O apoio financeiro para a realização deste trabalho foi concedido pela American Society of Pharmacognosy Foundation (1998 Research Starter Grant) e pela Fundação de Amparo à Pesquisa do Estado de São Paulo (96/043165 e 01/03095-5) a R. G. S. Berlinck; A. M. do Nascimento (03/ 10805-4), S. P. de Lira (03/06471-3) e M. H. Kossuga agradecem, respectivamente, à FAPESP e à CAPES pelas bolsas de estudos concedidas. R. G. S. Berlinck, E. Hajdu, R. M. Rocha e A. G. Ferreira também agradecem ao $\mathrm{CNPq}$ pelas bolsas de produtividade em pesquisa.

\section{Dados cristalográficos}

Os dados cristalográficos da estrutura da asterubina (10) foram depositados no Cambridge Crystallographic Data Center, CCDC No. 613063. Cópias destas informações podem ser obtidas com The Director, CCDC, 12 Union Road, Cambridge, CB2 1EZ, UK (e-mail: deposit@ccdc.cam.ac.uk ou http://www.ccdc.cam.ac.uk).

\section{REFERÊNCIAS}

1. Blunt, J. W.; Copp, B. R.; Munro, M. H. G.; Nat. Prod. Rep. 2006, 23, 26

2. Berlinck, R. G. S.; Hajdu, E.; Rocha, R. M.; Oliveira, J. H. H. L.; Hernandez, I. L. C.; Seleghim, M. H. R.; Granato, A. C.; Almeida, E. V. R.; Nunez, C. V.; Muricy, G.; Peixinho, S.; Pessoa, C; Moraes, M. O.; Cavalcanti, B. C.; Nascimento, G. G. F.; Thiemann, O.; Silva, M.; Souza, A. O.; Silva, C. L.; Minarini, P. R. R.; J. Nat. Prod. 2004, 67, 510.

3. Harbour, G. C.; Tymiak, A. A.; Rinehart Jr, K. L.; Shaw, P. D.; Hughes Jr, R. G.; Mizsak, S. A.; Coats, J. H.; Zurenko, G. E.; Li, L. H.; Kuentzel, S. L.; J. Am. Chem. Soc. 1981, 103, 5604.
4. Gallimore, W. A.; Kelly, M.; Scheuer, P. J.; J. Nat. Prod. 2005, 68, 1420.

5. Berlinck, R. G. S.; Kossuga, M. H.; Nat. Prod. Rep. 2005, 22, 516 e referências citadas.

6. van Soest, R. W. M.; Braekman, J. C.; Faulkner, D. J.; Hajdu, E.; Harper, M. K.; Vacelet, J.; Bull. Inst. R. Sci. Nat. Belg. 1996, 66 (suppl.), 89.

7. Lira, T. O.; Berlinck, R. G. S.; Nascimento, G. G. F.; Hajdu, E.; J. Braz. Chem. Soc. 2006, 17, 1233.

8. Pinheiro, U.; Dissertação de Mestrado, Universidade de São Paulo, Brasil, 2002.

9. Andersen, R. J.; Faulkner, D. J.; Tetrahedron Lett. 1973, 14, 1175.

10. Minale, L.; Sodano, G.; Chan, W. R.; Chen, A. M.; J. Chem. Soc., Chem. Comm. 1972, 11, 674.

11. Ciminiello, P.; Constantino, V.; Fattorusso, E.; Magno, S.; Mangoni, A.; Pansini, M.; J. Nat. Prod. 1994, 57, 705.

12. Ciminiello, P.; Dell'Aversano, C.; Fattorusso, E.; Magno, S.; Carrano, L.; Pansini, M.; Tetrahedron 1996, 52, 9863.

13. Aiello, A.; Fattorusso, E.; Menna, M.; Pansini, M.; Biochem. Syst. Ecol. 1995, 23, 377

14. Ogamino, T.; Nishiyama, S.; Tetrahedron 2003, 59, 9419.

15. Kossuga, M. H.; Macmillan, J. B.; Rorgers, E. W.; Molinski, T. F.; Nascimento, G. G. F.; Rocha, R. M.; Berlinck, R. G. S.; J. Nat. Prod. 2004 , 67, 1879.

16. Granato, A. C.; Oliveira, J. H. H. L.; Seleghim, M. H. R.; Berlinck, R. G. S.; Macedo, M. L.; Ferreira, A. G.; Rocha, R. M.; Hajdu, E.; Peixinho, S.; Pessoa, C.; Moraes, M. O.; Cavalcanti, B. C.; Quim. Nova 2005, $28,192$.

17. Fusetani, N.; Matsunaga, S.; Konosu, S.; Tetrahedron Lett. 1981, 22, 1985.

18. Kanazawa S.; Fusetani N.; Matsunaga S.; Tetrahedron 1992, 48, 5467.

19. Sperry, S.; Crews, P.; J. Nat. Prod. 1997, 60, 29.

20. Yang, S. W.; Buivich, A.; Chan, T. M.; Smith, M.; Lachowicz, J.; Pomponi, S. A.; Wright, A. E.; Mierzwa, R.; Patel, M.; Gullo, V.; Chu, M.; Bioorg. Med. Chem. Lett. 2003, 13, 1791.

21. Moni, R. W.; Willis, R. J.; Quinn, R. J.; Bioorg. Med. Chem. Lett. 1992, 2, 1631.

22. Slate, D. L.; Lee, R. H.; Rodriguez, J.; Crews, P.; Biochem. Biophys. Res. Commun. 1994, 203, 260.

23. Tsukamoto, S.; Kato, H.; Hirota, H.; Fusetani, N.; Biofouling 1997, 11, 283.

24. Kornprobst, J. M.; Sallenave, C.; Barnathan, G.; Comp. Biochem. Physiol. B-Biochem. Mol. Biol. 1998, 119, 1.

25. Gray, C.; Lira, S. P.; Silva, M.; Pimenta, E. F.; Thiemann, O. H.; Oliva, G.; Andersen, R. J.; Hajdu, E.; Berlinck, R. G. S.; J. Org. Chem. 2006, 71, 8685.

26. Davidson, B. S.; Chem. Rev. 1993, 93, 1771; Ding, Q.; Chichak, K.; Lown, J. W.; Curr. Med. Chem. 1999, 6, 1; Selvi, S. T.; Mohan, P. S.; Indian J. Chem, Sect. B: Org. Chem. Incl. Med. Chem. 1999, 38, 1118; Groundwater, P. W.; Munawar, M. A.; Adv. Heterocycl. Chem. 1998, 70, 89.

27. Ackermann, D.; Z. Physiol. Chem. 1935, 232, 206; Ackermann, D.; Verh. Physik.-Med. Ges. Wurzburg 1936, 59, 73.

28. Capon, R.; comunicação pessoal.

29. Lehninger, A. L.; Nelson, D. L.; Cox, M. M.; Princípios de Bioquímica, $2^{a}$ ed., Sarvier: São Paulo, 1995, p. 494-495.

30. Shen, Y.; Cheng, Y.; Lin, Y.; Guh, J.; Teng, C.; Ko, C.; J. Nat. Prod. 2004, $67,542$.

31. Lyanage, G. K.; Schmitz, F. J.; J. Nat. Prod. 1996, 59, 148.

32. Maia, L. F.; Epifanio, R. A.; Fenical, W.; J. Nat. Prod. 2000, 63, 1427.

33. Baker, B. J.; Okuda, R. K.; Yu, P. T. K.; Scheuer, P.J.; J. Am. Chem. Soc. 1985, 107, 2976.

34. Baker, B. J.; Scheuer, P. J.; J. Nat. Prod. 1994, 57, 1346.

35. Ross, R. A.; Scheuer, P. J.; Tetrahedron Lett. 1979, 4701.

36. Zapantis, A.; Lacy, M. K.; Horvat, R. T.; Grauer, D.; Barnes, B. J.; O’Neal, B.; Couldry, R.; J. Clin. Microbiol. 2005, 43, 2629.

37. Hjertstedt, J.; Hahn, B. L.; Kos, W. L.; Sohnle, P. G.; Mycoses 1998, 41, 487.

38. Aoki, S.; Naka, Y.; Itoh, T.; Furukawa, T.; Rachmat, R.; Akiyama, S.; Kobayashi, M.; Chem. Pharm. Bull. 2002, 50, 827.

39. North, A. C. T.; Phillips, D. C.; Mathews, F. S.; Acta Crystallogr., Sect. A: Found. Crystallogr. 1968, 24, 351.

40. Altomare, A.; Cascarano, G.; Giacovazzo, C.; Guagliardi, A.; J. Appl. Crystallogr. 1993, 26, 343.

41. Farrugia, L. J., J. Appl. Crystallogr. 1999, 32, 837.

42. Farrugia, L. J.; J. Appl. Crystallogr. 1997, 30, 565. 